\title{
EXISTENCE RESULTS FOR A CLASS OF SECOND ORDER DIFFERENTIAL EQUATIONS WITH NONLINEAR DERIVATIVE DEPENDENCE
}

\author{
SHAPOUR HEIDARKHANI ${ }^{1, *}$, JOHNNY HENDERSON ${ }^{2}$, AREZOO SOLIMANINIA ${ }^{1}$ \\ ${ }^{1}$ Department of Mathematics, Faculty of Sciences, Razi University, 67149 Kermanshah, Iran \\ ${ }^{2}$ Department of Mathematics, Baylor University, Waco, TX 76798-7328, USA
}

Abstract. We establish multiplicity results for nontrivial solutions of the quasilinear second order differential equation on a compact interval $[a, b] \subset \mathbb{R}$,

$$
\left\{\begin{array}{l}
-\left(p_{i}-1\right)\left|u_{i}^{\prime}(x)\right|^{p_{i}-2} u_{i}^{\prime \prime}(x)=\lambda F_{u_{i}}\left(x, u_{1}, \ldots, u_{n}\right) h_{i}\left(x, u_{i}^{\prime}\right), \quad x \in(a, b), \\
u_{i}(a)=u_{i}(b)=0
\end{array}\right.
$$

for $1 \leq i \leq n$, under appropriate hypotheses. Indeed, using a consequence of the local minimum theorem due to Bonanno and, the mountain pass theorem, we investigate the existence of two solutions for the problem under some algebraic conditions with the classical Ambrosett-Rabinowitz condition on the nonlinear terms. Moreover, by combining two algebraic conditions on the nonlinear terms, and employing two consequences of the local minimum theorem due to Bonanno, we guarantee the existence of two solutions for the scalar case of the problem. Applying the mountain pass theorem given by Pucci and Serrin, we ensure the existence of the third solution for our problem.

Keywords. Dirichlet problem; Nonlinear derivative dependence; Multiple solution; Variational method; Critical point theory.

2010 Mathematics Subject Classification. 34B15.

\section{INTRODUCTION}

In this paper, we study the following quasilinear system

$$
\left\{\begin{array}{l}
-\left(p_{i}-1\right)\left|u_{i}^{\prime}(x)\right|^{p_{i}-2} u_{i}^{\prime \prime}(x)=\lambda F_{u_{i}}\left(x, u_{1}, \ldots, u_{n}\right) h_{i}\left(x, u_{i}^{\prime}\right), \quad x \in(a, b), \\
u_{i}(a)=u_{i}(b)=0,
\end{array}\right.
$$

for $1 \leq i \leq n$, where $p_{i}>1$ for $1 \leq i \leq n, \lambda>0, a, b \in \mathbb{R}$ with $a<b, F:[a, b] \times \mathbb{R}^{n} \rightarrow \mathbb{R}$ is measurable with respect to $x$, for all $u \in \mathbb{R}^{n}$, continuously differentiable in $u$, for almost every $x \in[a, b]$, satisfies $\sup _{|\xi| \leq a}|F(\cdot, \xi)| \in L^{1}([a, b])$ for any $a>0, F(x, 0, \ldots, 0)=0$ for all $x \in[a, b], F_{t_{i}}$ is continuous in $[a, b] \times$

${ }^{*}$ Corresponding author.

E-mail addresses: s.heidarkhani@razi.ac.ir (S. Heidarkhani), Johnny_Henderson@baylor.edu (J. Henderson), a.solimaninia @ yahoo.com (A. Solimaninia).

Received March 6, 2018; Accepted June 4, 2018.

(C)2018 Journal of Nonlinear Functional Analysis 
$\mathbb{R}^{n}$ for $i=1, \ldots, n, h_{i}:[a, b] \times \mathbb{R} \rightarrow[0,+\infty)$ is bounded and continuous with $m_{i}:=\inf _{(x, t) \in[a, b] \times \mathbb{R}} h_{i}(x, t)>$ 0 for $1 \leq i \leq n$. Here, $F_{t_{i}}$ denotes the partial derivative of $F$ with respect to $t_{i}$.

The study of multiplicity of solutions is an important mathematical subject which is also interesting from the practical point of view because the physical processes described by boundary value problems for differential equations exhibit, generally, more than one solution. For example, certain chemical reactions in tubular reactors can be mathematically described by a nonlinear two-point boundary value problem with interest in seeing if multiple steady-states to the problem exist. For a treatment of chemical reactor theory and multiple solutions, we refer the readers to [1, Section 7] and references therein.

In recent years, many authors have studied the existence of nontrivial solutions for Laplacian systems and $p$-Laplacian systems; see $[2,3,4,5,6,7,8,9,10]$ and the references therein. Usually authors change the problem into a critical point problem of the corresponding energy functional and then apply critical point theory or variational methods. Or they change it into a fixed point problem of the corresponding compactly continuous mapping and then apply the topological degree theory or the method of lower and upper solutions. For instance, Costa and Magalhaes [3] unified the cooperative and noncooperative Laplacian systems, and they got the existence of nontrivial solutions via the variational approach. In [2] Conti, Terracini and Verzini dealt with a competitive Laplacian system and they established the existence of positive solutions by the Nehari procedure, critical point theory, and topological degree theory. In $[5,6]$, the authors studied the sublinear $p$-Laplacian systems, and they obtained the existence of positive solutions by the method of lower and upper solutions and Leray-Schauder degree theory, respectively. In [10], Zhang and Zhang considered the existence of nontrivial solutions for nonlinear Laplacian systems and $p$-Laplacian systems applying the direct variational method. Employing a recent three critical points theorem of Ricceri [11], the existence of at least three solutions for system (1.1) containing a perturbation term was discussed in [4], while in [7], the existence of at least three distinct solutions for the system (1.1) was established based on two critical points theorems.

The main features of this paper are as follows. First, using a consequence of the local minimum theorem due to Bonanno, and the mountain pass theorem, we discuss the existence of two nontrivial solutions in the space $W_{0}^{1, p_{1}}([a, b]) \times W_{0}^{1, p_{2}}([a, b]) \times \ldots \times W_{0}^{1, p_{n}}([a, b])$ for system (1.1) by combining an algebraic condition on $F$ with the classical Ambrosetti-Rabinowitz condition:

(AR): there exist $v>\max \left\{p_{i} ; 1 \leq i \leq n\right\}$ and $R>0$ such that

$$
0<v F(x, t) \leq \sum_{i=1}^{n} t_{i} F_{t_{i}}(x, t) \text { for all }|t| \geq R \text { and for all } x \in[a, b], t=\left(t_{1}, \ldots, t_{n}\right)
$$

The role of (AR) is to ensure the boundness of the Palais-Smale sequences for the Euler-Lagrange functional associated to the problem. This is very crucial in the applications of the critical point theory. Second, we obtain an existence result of three nontrivial solutions combining two algebraic conditions employing two consequences of the local minimum theorem due to Bonanno, which guarantees the existence of two local minima for the Euler-Lagrange functional, and applying the mountain pass theorem, as given by Pucci and Serrin (see [12]), to ensure the existence of the third critical point for the scaler case of the system.

For existence results for multiple solutions for second order differential equations with nonlinear derivative dependence, we refer to $[13,14,15,16]$ and the references therein. For more details on Theorem 
2.1, we refer the readers to [17, 18], where the theorem has already been applied to nonlinear secondorder differential equations problems. We also refer to [19,20,21] in which, Theorems 2.1 and 2.2 along with the mountain pass theorem have been successfully employed to ensure multiple solutions for some boundary value problems. We also refer the reader to the papers $[22,23]$ in which based on variational methods and critical point theory, the existence of multiple solutions for boundary value problems was discussed. For a thorough account on the subject, we also refer the readers to [24, 25].

\section{PRELIMINARIES}

Our main tools are Theorems 2.1 and 2.2 of this section, which are consequences of the existence result of a local minimum theorem for differentiable functionals [26, Theorem 3.1]. This is inspired by the Ricceri's variational principle (see [27]).

For a given non-empty set $X$, and two functionals $\Phi, \Psi: X \rightarrow \mathbb{R}$, we define the following functions

$$
\begin{gathered}
\vartheta\left(r_{1}, r_{2}\right)=\inf _{v \in \Phi^{-1}\left(r_{1}, r_{2}\right)} \frac{\sup _{u \in \Phi^{-1}\left(r_{1}, r_{2}\right)} \Psi(u)-\Psi(v)}{r_{2}-\Phi(v)}, \\
\rho_{1}\left(r_{1}, r_{2}\right)=\sup _{v \in \Phi^{-1}\left(r_{1}, r_{2}\right)} \frac{\Psi(v)-\sup _{u \in \Phi^{-1}\left(-\infty, r_{1}\right)} \Psi(u)}{\Phi(v)-r_{1}},
\end{gathered}
$$

for all $r_{1}, r_{2} \in \mathbb{R}, r_{1}<r_{2}$, and

$$
\rho_{2}(r)=\sup _{v \in \Phi^{-1}(r, \infty)} \frac{\Psi(v)-\sup _{u \in \Phi^{-1}(-\infty, r)} \Psi(u)}{\Phi(v)-r},
$$

for all $r \in \mathbb{R}$.

Theorem 2.1. [26, Theorem 5.1] Let $X$ be a real Banach space. Let $\Phi: X \rightarrow \mathbb{R}$ be a sequentially weakly lower semicontinuous, coercive, and continuously Gâteaux differentiable function whose Gâteaux derivative admits a continuous inverse on $X^{*}$. Let $\Psi: X \rightarrow \mathbb{R}$ be a continuously Gâteaux differentiable function whose Gâteaux derivative is compact. Assume that there are $r_{1}, r_{2} \in \mathbb{R}, r_{1}<r_{2}$, such that $\vartheta\left(r_{1}, r_{2}\right)<$ $\rho_{1}\left(r_{1}, r_{2}\right)$. Then, setting $I_{\lambda}:=\Phi-\lambda \Psi$, for each $\lambda \in\left(\frac{1}{\rho_{1}\left(r_{1}, r_{2}\right)}, \frac{1}{\vartheta\left(r_{1}, r_{2}\right)}\right)$, there is $u_{0, \lambda} \in \Phi^{-1}\left(r_{1}, r_{2}\right)$ such that $I_{\lambda}\left(u_{0, \lambda}\right) \leq I_{\lambda}(u), \forall u \in \Phi^{-1}\left(r_{1}, r_{2}\right)$ and $I_{\lambda}^{\prime}\left(u_{0, \lambda}\right)=0$.

Theorem 2.2. [26, Theorem 5.3] Let $X$ be a real Banach space. Let $\Phi: X \rightarrow \mathbb{R}$ be a continuously Gâteaux differentiable function whose Gâteaux derivative admits a continuous inverse on $X^{*}$. Let $\Psi: X \rightarrow \mathbb{R}$ be a continuously Gâteaux differentiable function whose Gâteaux derivative is compact. Fix $\inf _{X} \Phi<r<$ $\sup _{X} \Phi$ and assume that $\rho_{2}(r)>0$, and for each $\lambda>\frac{1}{\rho_{2}(r)}$, the functional $I_{\lambda}:=\Phi-\lambda \Psi$ is coercive. Then, for each $\lambda \in\left(\frac{1}{\rho_{2}(r)},+\infty\right)$, there is $u_{0, \lambda} \in \Phi^{-1}(r,+\infty)$ such that $I_{\lambda}\left(u_{0, \lambda}\right) \leq I_{\lambda}(u), \forall u \in \Phi^{-1}(r,+\infty)$ and $I_{\lambda}^{\prime}\left(u_{0, \lambda}\right)=0$.

\section{MAIN RESULTS}

Let $X$ be the Cartesian product of $n$ Sobolev spaces $W_{0}^{1, p_{1}}([a, b]), W_{0}^{1, p_{2}}([a, b]), \ldots$, and $W_{0}^{1, p_{n}}([a, b])$, i.e., $X=W_{0}^{1, p_{1}}([a, b]) \times W_{0}^{1, p_{2}}([a, b]) \times \ldots \times W_{0}^{1, p_{n}}([a, b])$, equipped with the norm

$$
\left\|\left(u_{1}, u_{2}, \ldots, u_{n}\right)\right\|=\sum_{i=1}^{n}\left\|u_{i}^{\prime}\right\|_{p_{i}},
$$


where

$$
\left\|u_{i}^{\prime}\right\|_{p_{i}}=\left(\int_{a}^{b}\left|u_{i}^{\prime}(x)\right|^{p_{i}} d x\right)^{1 / p_{i}}, i=1, \ldots, n .
$$

Since $p_{i}>1$ for $i=1, \ldots, n, X$ is compactly embedded in $(C([a, b]))^{n}$. By a classical solution of system (1.1), we mean a function $u=\left(u_{1}, \ldots, u_{n}\right)$ such that, for $i=1, \ldots, n, u_{i} \in C^{1}[a, b], u_{i}^{\prime} \in A C[a, b]$, and $u_{i}(x)$ satisfies (1.1) a.e. on $[a, b]$. We say that a function $u=\left(u_{1}, \ldots, u_{n}\right) \in X$ is a weak solution of system (1.1) if

$$
\sum_{i=1}^{n} \int_{a}^{b}\left(\int_{0}^{u_{i}^{\prime}(x)} \frac{\left(p_{i}-1\right)|\tau|^{p_{i}-2}}{h_{i}(x, \tau)} d \tau\right) v_{i}^{\prime}(x) d x-\lambda \int_{a}^{b} \sum_{i=1}^{n} F_{u_{i}}\left(x, u_{1}(x), \ldots, u_{n}(x)\right) v_{i}(x) d x=0
$$

for every $v=\left(v_{1}, \ldots, v_{n}\right) \in X$. Using standard methods, we see that a weak solution of (1.1) is indeed a classical solution (see [4, Lemma 2.2]).

In the sequel, let

$$
\begin{gathered}
\underline{p}=\min \left\{p_{i} ; 1 \leq i \leq n\right\}, \\
\bar{p}=\max \left\{p_{i} ; 1 \leq i \leq n\right\}, \\
m_{i}:=\inf _{(x, t) \in[a, b] \times \mathbb{R}} h_{i}(x, t)>0, \text { for } 1 \leq i \leq n, \\
M_{i}:=\sup _{(x, t) \in[a, b] \times \mathbb{R}} h_{i}(x, t), \text { for } 1 \leq i \leq n, \\
\bar{M}:=\max \left\{M_{i} ; 1 \leq i \leq n\right\}
\end{gathered}
$$

and

$$
\underline{M}:=\min \left\{m_{i} ; 1 \leq i \leq n\right\} .
$$

Then, $\bar{M} \geq M_{i} \geq m_{i} \geq \underline{M}>0$ for each $i=1, \ldots, n$. Put

$$
H_{i}(x, t)=\int_{0}^{t}\left(\int_{0}^{\tau} \frac{\left(p_{i}-1\right)|\delta|^{p_{i}-2}}{h_{i}(x, \delta)} d \delta\right) d \tau
$$

for $1 \leq i \leq n$ and for all $(x, t) \in[a, b] \times \mathbb{R}$. For all $\vartheta>0$, we denote by $Q(\vartheta)$ the set

$$
\left\{\left(t_{1}, \ldots, t_{n}\right) \in \mathbb{R}^{n}: \sum_{i=1}^{n} \frac{\left|t_{i}\right|^{p_{i}}}{p_{i}} \leq \vartheta\right\}
$$

For a given non-negative constant $c$ and a given positive constant $d$ with $\frac{2^{p} c}{(b-a)^{p^{*}-1} \bar{p} \bar{M}} \neq D_{1}(d)$, put

$$
a_{d}(c):=\frac{\int_{a}^{b} \sup _{\left(t_{1}, \ldots, t_{n}\right) \in Q(c)} F\left(x, t_{1}, \ldots, t_{n}\right) d x-\int_{a+\alpha}^{b-\beta} F(x, d, \ldots, d) d x}{\frac{2^{\underline{p}} c}{(b-a)^{p^{*}-1} \bar{p} \bar{M}}-D_{1}(d)},
$$

where

and

$$
p^{*}= \begin{cases}\bar{p} & \text { if } b-a \geq 1 \\ \underline{p} & \text { if } 0<b-a<1\end{cases}
$$

$$
D_{1}(d):=\sum_{i=1}^{n}\left(\int_{a}^{a+\alpha} H_{i}\left(x, \frac{d\left(p_{i}-1\right)(x-a)^{p_{i}-2}}{\alpha^{p_{i}-1}}\right) d x+\int_{b-\beta}^{b} H_{i}\left(x,-\frac{d\left(p_{i}-1\right)(b-x)^{p_{i}-2}}{\beta^{p_{i}-1}}\right) d x\right) .
$$

We now formulate our main result as follows.

Theorem 3.1. Suppose either $p \geq 2$ or $\bar{p}<2$. Assume that there exist a nonnegative constant $c_{1}$ and four positive constants $\alpha, \beta, c_{2}$ and $d$ with $\alpha+\beta<b-a$ and $c_{1}<\frac{(b-a)^{p^{*}-1} \bar{p} \bar{M}}{2^{\underline{p}}} D_{1}(d)<c_{2}$ such that

$\left(\mathrm{a}_{1}\right) \quad F\left(x, t_{1}, \ldots, t_{n}\right) \geq 0$ for each $\left(x, t_{1}, \ldots, t_{n}\right) \in([a, a+\alpha] \cup[b-\beta, b]) \times[0, d]^{n} ;$ 
$\left(\mathrm{a}_{2}\right)$

$$
\begin{array}{ll}
\left(\mathrm{a}_{2}\right) & a_{d}\left(c_{2}\right)<a_{d}\left(c_{1}\right) ; \\
\left(\mathrm{a}_{3}\right) & F_{t_{i}}(x, 0, \cdots, 0) \neq 0,1 \leq i \leq n \text { for all } x \in[a, b] \text { and there exist } v>\bar{p} \text { and } R>0 \text { such that } \\
& 0<v F(x, t) \leq \sum_{i=1}^{n} t_{i} F_{t_{i}}(x, t) \text { for all }|t| \geq R \text { and for all } x \in[a, b], t=\left(t_{1}, \ldots, t_{n}\right) .
\end{array}
$$

Then, for each $\lambda \in\left(\frac{1}{a_{d}\left(c_{1}\right)}, \frac{1}{a_{d}\left(c_{2}\right)}\right)$, system (1.1) admits at least two nontrivial classical solutions $u^{*}$ and $u^{* *}$, with $u^{*}$ such that

$$
\frac{2^{\underline{p}} c_{1}}{(b-a)^{p^{*}-1} \bar{p} \bar{M}}<\sum_{i=1}^{n}\left(\int_{a}^{a+\alpha} H_{i}\left(x, u^{*}(x)\right) d x+\int_{b-\beta}^{b} H_{i}\left(x, u^{*}(x)\right) d x\right)<\frac{2^{\underline{p}} c_{2}}{(b-a)^{p^{*}-1} \bar{p} \bar{M}} .
$$

Proof. We will apply Theorem 2.1. To this end, take the functionals $\Phi, \Psi: X \rightarrow \mathbb{R}$ defined by

$$
\Phi(u)=\sum_{i=1}^{n} \int_{a}^{b} H_{i}\left(x, u_{i}^{\prime}(x)\right) d x
$$

and

$$
\Psi(u)=\int_{a}^{b} F\left(x, u_{1}(x), \ldots, u_{n}(x)\right) d x
$$

for each $u=\left(u_{1}, \ldots, u_{n}\right) \in X$. It is well known that $\Psi$ is a Gâteaux differentiable functional and sequentially weakly lower semicontinuous whose Gâteaux derivative at the point $u \in X$ is the functional $\Psi^{\prime}(u) \in X^{*}$ given by

$$
\Psi^{\prime}(u)(v)=\int_{a}^{b} \sum_{i=1}^{n} F_{u_{i}}\left(x, u_{1}(x), \ldots, u_{n}(x)\right) v_{i}(x) d x
$$

for every $v=\left(v_{1}, \ldots, v_{n}\right) \in X$, and $\Psi^{\prime}: X \rightarrow X^{*}$ is a compact operator. Moreover, $\Phi$ is a Gâteaux differentiable functional whose Gâteaux derivative at the point $u \in X$ is the functional $\Phi^{\prime}(u) \in X^{*}$ given by

$$
\Phi^{\prime}(u)(v)=\sum_{i=1}^{n} \int_{a}^{b}\left(\int_{0}^{u_{i}^{\prime}(x)} \frac{\left(p_{i}-1\right)|\tau|^{p_{i}-2}}{h_{i}(x, \tau)} d \tau\right) v_{i}^{\prime}(x) d x
$$

for every $v=\left(v_{1}, \ldots, v_{n}\right) \in X$. Furthermore, Lemma 2.1 of [4] implies $\left(\Phi^{\prime}\right)^{-1}: X^{*} \rightarrow X$ exists and is continuous, and since $\Phi^{\prime}$ is monotone, $\Phi$ is sequentially weakly lower semicontinuous. Choose $r_{1}=$ $\frac{2^{\underline{p}} c_{1}}{(b-a)^{p^{*}-1} \bar{p} \bar{M}}, r_{2}=\frac{2^{\underline{p}} c_{2}}{(b-a)^{p^{*}-1} \bar{p} \bar{M}}$ and $w=\left(w_{1}, \ldots, w_{n}\right)$ with

$$
w_{i}(x)= \begin{cases}\frac{1}{\alpha^{p_{i}-1}} d(x-a)^{p_{i}-1} & \text { if } a \leq x<a+\alpha, \\ d & \text { if } a+\alpha \leq x \leq b-\beta, \\ \frac{1}{\beta^{p^{-1}}} d(b-x)^{p_{i}-1} & \text { if } b-\beta<x \leq b,\end{cases}
$$

for $1 \leq i \leq n$. From (3.2) and the condition $c_{1}<\frac{(b-a)^{p^{*}-1} \bar{p} \bar{M}}{2^{\underline{p}}} D_{1}(d)<c_{2}$, we obtain $r_{1}<\Phi\left(u_{1}\right)<r_{2}$. Moreover, taking into account that $0<\underline{M} \leq h_{i}(x, t) \leq \bar{M}$ for each $(x, t) \in[a, b] \times \mathbb{R}$ and for $i=1, \ldots, n$, we see from (3.2) that

$$
\frac{1}{\bar{M}} \sum_{i=1}^{n} \frac{\left\|u_{i}^{\prime}\right\|_{p_{i}}^{p_{i}}}{p_{i}} \leq \Phi(u) \leq \frac{1}{\underline{M}} \sum_{i=1}^{n} \frac{\left\|u_{i}^{\prime}\right\|_{p_{i}}^{p_{i}}}{p_{i}} \text { for all } u=\left(u_{1}, \ldots, u_{n}\right) \in X
$$

Since for $1 \leq i \leq n$,

$$
\max _{x \in[a, b]}\left|u_{i}(x)\right| \leq \frac{(b-a)^{\frac{p_{i}-1}{p_{i}}}}{2}|| u_{i}^{\prime} \|_{p_{i}}
$$


for each $u_{i} \in W_{0}^{1, p_{i}}([a, b])$ (see [28]), we have

$$
\max _{x \in[a, b]} \sum_{i=1}^{n} \frac{\left|u_{i}(x)\right|^{p_{i}}}{p_{i}} \leq \frac{(b-a)^{p^{*}-1}}{2^{\underline{p}}} \sum_{i=1}^{n} \frac{\left\|u_{i}^{\prime}\right\|_{p_{i}}^{p_{i}}}{p_{i}}
$$

for each $u=\left(u_{1}, u_{2}, \ldots, u_{n}\right) \in X$. This, for each $r_{2}>0$, along with (3.5), ensures that

$$
\Phi^{-1}(]-\infty, r_{2}[) \subseteq\left\{u \in X ; \max \sum_{i=1}^{n} \frac{\left|u_{i}(x)\right|^{p_{i}}}{p_{i}} \leq c_{2} \text { for each } x \in[a, b]\right\},
$$

and it follows that

$$
\sup _{u \in \Phi^{-1}(]-\infty, r_{2}[)} \Psi(u) \leq \int_{a}^{b} \sup _{\left(t_{1}, \ldots, t_{n}\right) \in Q\left(c_{2}\right)} F\left(x, t_{1}, \ldots, t_{n}\right) d x .
$$

Moreover, since $0 \leq w_{i}(x) \leq d$ for each $x \in[a, b]$ and for $1 \leq i \leq n$, the assumption $\left(\mathrm{a}_{1}\right)$ ensures that

$$
\begin{gathered}
\int_{a}^{a+\alpha} F\left(x, \frac{1}{\alpha^{p_{1}-1}} d(x-a)^{p_{1}-1}, \ldots, \frac{1}{\alpha^{p_{n}-1}} d(x-a)^{p_{n}-1}\right) d x \\
+\int_{b-\beta}^{b} F\left(x, \frac{1}{\beta^{p_{1}-1}} d(b-x)^{p_{1}-1}, \ldots, \frac{1}{\beta^{p_{n}-1}} d(b-x)^{p_{n}-1}\right) d x \geq 0 .
\end{gathered}
$$

So,

$$
\Psi(w) \geq \int_{a+\alpha}^{b-\beta} F(x, d, \ldots, d) d x
$$

It follows that

$$
\begin{aligned}
\vartheta\left(r_{1}, r_{2}\right) & \leq \frac{\sup _{u \in \Phi^{-1}(]-\infty, r_{2}[)} \Psi(u)-\Psi(w)}{r_{2}-\Phi(w)} \\
& \leq \frac{\int_{a}^{b} \sup _{\left(t_{1}, \ldots, t_{n}\right) \in Q\left(c_{2}\right)} F\left(x, t_{1}, \ldots, t_{n}\right) d x-\Psi(w)}{r_{2}-\Phi(w)} \\
& \leq \frac{\int_{a}^{b} \sup _{\left(t_{1}, \ldots, t_{n}\right) \in Q\left(c_{2}\right)} F\left(x, t_{1}, \ldots, t_{n}\right) d x-\int_{a+\alpha}^{b-\beta} F(x, d, \ldots, d) d x}{\frac{2^{\underline{\underline{p}} c_{2}}}{(b-a)^{p^{*}-1} \bar{p} \bar{M}}-D_{1}(d)} \\
& =a_{d}\left(c_{2}\right) .
\end{aligned}
$$

On the other hand, by the same reasoning as before, one has

$$
\begin{aligned}
\rho_{1}\left(r_{1}, r_{2}\right) & \geq \frac{\Phi(w)-\sup _{u \in \Phi^{-1}(]-\infty, r_{1}[)} \Psi(u)}{\Phi(w)-r_{1}} \\
& \geq \frac{\Psi(w)-\int_{a}^{b} \sup _{\left(t_{1}, \ldots, t_{n}\right) \in Q\left(c_{1}\right)} F\left(x, t_{1}, \ldots, t_{n}\right) d x}{\Phi(w)-r_{1}} \\
& \geq \frac{\int_{a}^{b} \sup _{\left(t_{1}, \ldots, t_{n}\right) \in Q\left(c_{1}\right)} F\left(x, t_{1}, \ldots, t_{n}\right) d x-\int_{a+\alpha}^{b-\beta} F(x, d, \ldots, d) d x}{\frac{2^{\underline{\underline{p}}} c_{1}}{(b-a)^{p^{*}-1} \bar{p} \bar{M}}-D_{1}(d)} \\
& =a_{d}\left(c_{1}\right) .
\end{aligned}
$$

Therefore, from Assumption $\left(\mathrm{a}_{2}\right)$ one has

$$
\vartheta\left(r_{1}, r_{2}\right)<\rho_{1}\left(r_{1}, r_{2}\right)
$$


Hence, applying Theorem 2.1, for each $\lambda \in\left(\frac{1}{a_{d}\left(c_{1}\right)}, \frac{1}{a_{d}\left(c_{2}\right)}\right)$, the functional $\Phi-\lambda \Psi$ admits at least one critical point $u^{*}$ which is a local minimum such that $r_{1}<\Phi\left(u^{*}\right)<r_{2}$, that is,

$$
\frac{2^{\underline{p}} c_{1}}{(b-a)^{p^{*}-1} \bar{p} \bar{M}}<\sum_{i=1}^{n}\left(\int_{a}^{a+\alpha} H_{i}\left(x, u^{*}(x)\right) d x+\int_{b-\beta}^{b} H_{i}\left(x, u^{*}(x)\right) d x\right)<\frac{2^{\underline{p}} c_{2}}{(b-a)^{p^{*}-1} \bar{p} \bar{M}} .
$$

Now, we prove the existence of the second local minimum distinct from the first one. To this end, we must show that the functional $\Phi-\lambda \Psi$ satisfies the hypotheses of the mountain pass theorem. Clearly, the functional $\Phi-\lambda \Psi$ is of class $C^{1}$ and $(\Phi-\lambda \Psi)(0)=0$. From the first part of the proof, we can assume that $u^{*}$ is a strict local minimum for $\Phi-\lambda \Psi$ in $X$. Therefore, there is $\rho>0$ such that

$$
\inf _{\left\|u-u^{*}\right\|=\rho}(\Phi-\lambda \Psi)(u)>(\Phi-\lambda \Psi)\left(u^{*}\right) .
$$

Now, choosing any $u \in X \backslash\{0\}$, from (3.5) and ( $\left.\mathrm{a}_{3}\right)$, one has

$$
\begin{aligned}
& (\Phi-\lambda \Psi)(t u) \leq \frac{1}{\underline{M}} \sum_{i=1}^{n} \frac{\|\left. t u_{i}^{\prime}\right|_{p_{i}} ^{p_{i}}}{p_{i}}-\lambda \int_{a}^{b} F\left(x, t u_{1}(x), \ldots, t u_{n}(x)\right) d x \\
& \leq \frac{1}{\underline{M}_{i=1}} \sum_{i=1}^{n} \frac{t^{p_{i}} \| u_{i}^{\prime}||_{p_{i}}^{p_{i}}}{p_{i}}-\lambda C_{1} t^{v} \sum_{i=1}^{n} \int_{a}^{b}\left|u_{i}(x)\right|^{v} d x+C_{2}(b-a) \longrightarrow-\infty
\end{aligned}
$$

as $t \rightarrow+\infty$ for some $C_{1}, C_{2}>0$, since $v>\bar{p}$. So the condition [29, $\left(I_{2}\right)$, Theorem 2.2] is verified. Moreover, standard computations show that $\Phi-\lambda \Psi$ satisfies the (P-S) condition. Hence, the classical theorem of Ambrosetti and Rabinowitz ensures a critical point $u^{* *}$ of $\Phi-\lambda \Psi$ such that

$$
(\Phi-\lambda \Psi)\left(u^{* *}\right)>(\Phi-\lambda \Psi)\left(u^{*}\right)
$$

Moreover, since $F_{t_{i}}(x, 0, \cdots, 0) \neq 0,1 \leq i \leq n$ for all $x \in[a, b]$, we see that $u \equiv 0$ is not a solution of (1.1), so $u^{* *}$ is nontrivial. Since the critical points of the functional $\Phi-\lambda \Psi$ are classical solutions of (1.1), we find the desired result. This completes the proof.

The following result is an immediate consequence of Theorem 3.1.

Corollary 3.2. Suppose either $\underline{p} \geq 2$ or $\bar{p}<2$. Assume that there exist two positive constants $c$ and $d$ with $D_{1}(d)<\frac{2^{\underline{p}} c}{(b-a)^{p^{*}-1} \bar{p} \bar{M}}$ such that Assumptions $\left(a_{1}\right)$ and $\left(a_{3}\right)$ in Theorem 3.1 hold. Furthermore, suppose that

$$
\frac{\int_{a}^{b} \sup _{\left(t_{1}, \ldots, t_{n}\right) \in Q(c)} F\left(x, t_{1}, \ldots, t_{n}\right) d x}{c}<\frac{2 \underline{p}}{(b-a)^{p^{*}-1} \bar{p} \bar{M}} \frac{\int_{a+\alpha}^{b-\beta} F(x, d, \ldots, d) d x}{D_{1}(d)} .
$$

Then, for every $\lambda \in\left(\frac{D_{1}(d)}{\int_{a+\alpha}^{b-\beta} F(x, d, \ldots, d) d x}, \frac{\frac{2 \underline{p}_{c}}{(b-a) p^{\underline{p}}-1_{\bar{p}} \bar{M}}}{\int_{a}^{b} \sup _{\left(t_{1}, \ldots, t_{n}\right) \in Q(c)} F\left(x, t_{1}, \ldots, t_{n}\right) d x x}\right)$, system (1.1) admits at least two nontrivial classical solutions $u^{*}$ and $u^{* *}$, with $u^{*}$ such that

$$
0<\sum_{i=1}^{n}\left(\int_{a}^{a+\alpha} H_{i}\left(x, u^{*}\right) d x+\int_{b-\beta}^{b} H_{i}\left(x, u^{*}\right) d x\right)<\frac{2^{\underline{p}} c}{(b-a)^{p^{*}-1} \bar{p} \bar{M}} .
$$


Proof. The conclusion follows from Theorem 3.1 by taking $c_{1}=0$ and $c_{2}=c$. Indeed, from the assumptions, one has

$$
\begin{aligned}
a_{d}(c) & <\frac{\left(1-\frac{D_{1}(d)}{\frac{2^{\underline{\underline{p}} c}}{(b-a)^{p^{*}}-1} \overline{\bar{p}}}\right) \int_{a}^{b} \sup _{\left(t_{1}, \ldots, t_{n}\right) \in Q(c)} F\left(x, t_{1}, \ldots, t_{n}\right) d x}{\frac{2^{\underline{\underline{p}} c}}{(b-a)^{p^{*}-1} \bar{p} \bar{M}}-D_{1}(d)} \\
& =\frac{\int_{a}^{b} \sup _{\left(t_{1}, \ldots, t_{n}\right) \in Q(c)} F\left(x, t_{1}, \ldots, t_{n}\right) d x}{\frac{2^{\underline{p}} c}{(b-a)^{p^{*}-1} \bar{p}}} \\
& <\frac{\int_{a+\alpha}^{b-\beta} F(x, d, \ldots, d) d x}{D_{1}(d)} \\
& =a_{d}(0) .
\end{aligned}
$$

In particular, one has

$$
a_{d}(c)<\frac{\int_{a}^{b} \sup _{\left(t_{1}, \ldots, t_{n}\right) \in Q(c)} F\left(x, t_{1}, \ldots, t_{n}\right) d x}{D_{1}(d)} .
$$

Hence, Theorem 3.1 ensures the result.

Now, we present an application of Theorem 2.2 which we will use to obtain multiple solutions.

Theorem 3.3. Suppose either $\underline{p} \geq 2$ or $\bar{p}<2$. Assume that there exist two positive constants $\bar{c}$ and $\bar{d}$ with $\frac{2^{\underline{p}} \bar{c}}{(b-a)^{p^{*}-1} \bar{p} \bar{M}}<D_{1}(\bar{d})$ such that

$\left(\mathrm{b}_{1}\right) \quad F\left(x, t_{1}, \ldots, t_{n}\right) \geq 0$ for each $\left(x, t_{1}, \ldots, t_{n}\right) \in([a, a+\alpha] \cup[b-\beta, b]) \times[0, \bar{d}]^{n} ;$

(b $\left.\mathrm{b}_{2}\right) \quad \int_{a}^{b} \sup _{\left(t_{1}, \ldots, t_{n}\right) \in Q(\bar{c})} F\left(x, t_{1}, \ldots, t_{n}\right) d x<\int_{a+\alpha}^{b-\beta} F(x, \bar{d}, \ldots, \bar{d}) d x$;

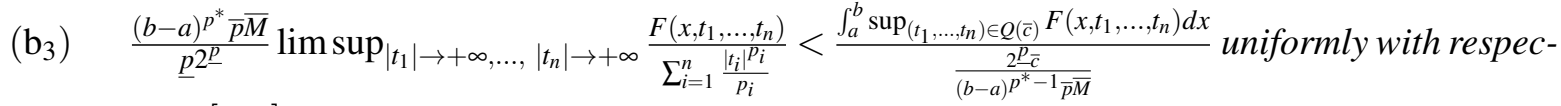
t to $x \in[a, b]$.

Then, for each $\lambda>\bar{\lambda}$, where

$$
\bar{\lambda}=\frac{D_{1}(\bar{d})-\frac{2^{\frac{p}{\bar{c}}}}{(b-a)^{p^{*}-1} \bar{p} \bar{M}}}{\int_{a+\alpha}^{b-\beta} F(x, \bar{d}, \ldots, \bar{d}) d x-\int_{a}^{b} \sup _{\left(t_{1}, \ldots, t_{n}\right) \in Q(\bar{c})} F\left(x, t_{1}, \ldots, t_{n}\right) d x},
$$

system (1.1) admits at least one nontrivial classical solution $\bar{u}$ such that

$$
\sum_{i=1}^{n}\left(\int_{a}^{a+\alpha} H_{i}(x, \bar{u}(x)) d x+\int_{b-\beta}^{b} H_{i}(x, \bar{u}(x)) d x\right)>\frac{2^{\underline{p}} \bar{c}}{(b-a)^{p^{*}-1} \bar{p} \bar{M}} .
$$

Proof. Let $\Phi$ and $\Psi$ be as in (3.2) and (3.3), respectively. Let us employ Theorem 2.2 to our functionals. The functionals $\Phi$ and $\Psi$ satisfy all regularity assumptions required in Theorem 2.2. Moreover, from $\left(b_{3}\right)$ there exist two constants $\gamma, \eta \in \mathbb{R}$ with

$$
0<\gamma<\frac{\int_{a}^{b} \sup _{\left(t_{1}, \ldots, t_{n}\right) \in Q(\bar{c})} F\left(x, t_{1}, \ldots, t_{n}\right) d x}{\frac{22_{\bar{c}} \bar{c}}{(b-a)^{p^{*}-1} \bar{p} \bar{M}}},
$$

such that

$$
\frac{(b-a)^{p^{*}} \bar{p} \bar{M}}{\underline{p}^{\underline{p}}} F\left(x, t_{1}, \ldots, t_{n}\right) \leq \gamma \sum_{i=1}^{n} \frac{\left|t_{i}\right|^{p_{i}}}{p_{i}}+\eta \text { for all } x \in[a, b] \text { and for all }\left(t_{1}, \ldots, t_{n}\right) \in \mathbb{R}^{n} .
$$


Fix $u=\left(u_{1}, \ldots, u_{n}\right) \in X$. Then

$$
F\left(x, u_{1}(x), \ldots, u_{n}(x)\right) \leq \frac{\underline{p} 2^{\underline{p}}}{(b-a)^{p^{*}} \bar{p} \bar{M}}\left(\gamma \sum_{i=1}^{n} \frac{\left|u_{i}(x)\right|^{p_{i}}}{p_{i}}+\eta\right) \text { for a.e. } x \in[a, b] .
$$

So, for any fixed $\lambda>\bar{\lambda}$, from (3.2), (3.3), (3.5), (3.6) and (3.7), we have

$$
\begin{aligned}
& \Phi(u)-\lambda \Psi(u)=\sum_{i=1}^{n} \int_{a}^{b} H_{i}\left(x, u_{i}^{\prime}(x)\right) d x-\lambda \int_{a}^{b} F\left(x, u_{1}(x), \ldots, u_{n}(x)\right) d x \\
& \geq \frac{1}{\bar{p} \bar{M}} \sum_{i=1}^{n} \|\left. u_{i}^{\prime}\right|_{p_{i}} ^{p_{i}}-\lambda \gamma \frac{\underline{p} 2^{\underline{p}}}{(b-a)^{p^{*}} \overline{\bar{p}} \bar{M}} \sum_{i=1}^{n} \int_{a}^{b} \frac{\left|u_{i}(x)\right|^{p_{i}}}{p_{i}} d x-\lambda \frac{\underline{p} 2^{\underline{p}}}{(b-a)^{\bar{p}-1} \overline{\bar{p}} \bar{M}} \eta \\
& \geq \frac{1}{\bar{p} \bar{M}} \sum_{i=1}^{n}\left\|u_{i}^{\prime}\right\|_{p_{i}}^{p_{i}}-\lambda \gamma \frac{\underline{p} 2^{\underline{p}}}{(b-a)^{p^{*}} \bar{p} \bar{M}} \frac{(b-a)^{p^{*}}}{\underline{p} 2^{\underline{p}}} \sum_{i=1}^{n}\left\|u_{i}^{\prime}\right\|_{p_{i}}^{p_{i}}-\lambda \frac{\underline{p} 2^{\underline{p}}}{(b-a)^{\bar{p}-1} \bar{p} \bar{M}} \eta \\
& =\frac{1}{\bar{p} \bar{M}}(1-\lambda \gamma) \sum_{i=1}^{n}\left\|u_{i}^{\prime}\right\|_{p_{i}}^{p_{i}}-\lambda \frac{\underline{p} 2^{2}}{(b-a)^{\bar{p}}-1 \bar{p} \bar{M}} \eta \\
& \geq \frac{1}{\bar{p} \bar{M}}\left(1-\frac{\gamma_{(b-a)^{p^{*}-1} \bar{p} \bar{M}}}{\int_{a}^{b} \sup _{\left(t_{1}, \ldots, t_{n}\right) \in Q(\bar{c})} F\left(x, t_{1}, \ldots, t_{n}\right) d x}\right) \sum_{i=1}^{n}\left\|u_{i}^{\prime}\right\|_{p_{i}}^{p_{i}} \\
& -\lambda \frac{\underline{p} 2^{\underline{p}}}{(b-a)^{\bar{p}-1} \bar{p} \bar{M}} \eta \text {. }
\end{aligned}
$$

Thus

$$
\lim _{\|u\| \rightarrow+\infty}(\Phi(u)-\lambda \Psi(u))=+\infty,
$$

which means the functional $\Phi-\lambda \Psi$ is coercive. Put

$$
\bar{r}=\frac{2^{\underline{p}} \bar{c}}{(b-a)^{p^{*}-1} \bar{p} \bar{M}}
$$

and $w=\left(w_{1}, \ldots, w_{n}\right)$ with

$$
w_{i}(x)= \begin{cases}\frac{1}{\alpha^{p_{i}-1}} \bar{d}(x-a)^{p_{i}-1} & \text { if } a \leq x<a+\alpha, \\ \frac{1}{\beta^{p_{i}-1}} \bar{d}(b-x)^{p_{i}-1} & \text { if } a+\alpha \leq x \leq b-\beta, \\ \text { if } b-\beta<x \leq b .\end{cases}
$$

Arguing as in the proof of Theorem 3.1, we obtain that

$$
\rho_{2}(\bar{r}) \geq \frac{\int_{a+\alpha}^{b-\beta} F(x, \bar{d}, \ldots, \bar{d}) d x-\int_{a}^{b} \sup _{\left(t_{1}, \ldots, t_{n}\right) \in Q(\bar{c})} F\left(x, t_{1}, \ldots, t_{n}\right) d x}{\frac{2^{\underline{p}} \bar{c}}{(b-a)^{p^{-}-1} \bar{p} \bar{M}}-D_{1}(\bar{d})} .
$$

So, from our assumptions, it follows that $\rho_{2}(\bar{r})>0$. Hence, Theorem 2.2 ensures that the functional $\Phi-\lambda \Psi$ admits at least one local minimum $\bar{u} \in X$ such that

$$
\sum_{i=1}^{n}\left(\int_{a}^{a+\alpha} H_{i}(x, \bar{u}(x)) d x+\int_{b-\beta}^{b} H_{i}(x, \bar{u}(x)) d x\right)>\frac{2^{p} \bar{c}}{(b-a)^{p^{*}-1} \bar{p} \bar{M}} .
$$

Since the critical points of the functional $\Phi-\lambda \Psi$ are classical solutions of (1.1), we have the desired conclusion.

As an application of the results, we consider the problem

$$
\left\{\begin{array}{l}
-(p-1)\left|u^{\prime}(x)\right|^{p-2} u^{\prime \prime}(x)=\lambda \alpha(x) g(u) h\left(u^{\prime}\right), \quad x \in(a, b), \\
u(a)=u(b)=0
\end{array}\right.
$$


where $p>1, \lambda>0, \alpha \in L^{1}([a, b])$ such that $\alpha(x) \geq 0$ a.e. $x \in[a, b], \alpha \neq \equiv, g: \mathbb{R} \longrightarrow \mathbb{R}$ is a nonnegative continuous function and $h: \mathbb{R} \rightarrow] 0,+\infty\left[\right.$ is a bounded and continuous function with $m:=\inf _{t \in \mathbb{R}} h(t)>0$ and $M:=\sup _{t \in \mathbb{R}} h(t)$.

We introduce the functions $G: \mathbb{R} \rightarrow \mathbb{R}$ and $H: \mathbb{R} \rightarrow \mathbb{R}$, respectively, as follows

$$
G(t)=\int_{0}^{t} g(\xi) d \xi \text { for all } t \in \mathbb{R}
$$

and

for all $t \in \mathbb{R}$.

$$
H(t)=\int_{0}^{t}\left(\int_{0}^{\tau} \frac{(p-1)|\delta|^{p-2}}{h(\delta)} d \delta\right) d \tau
$$

Now, we point out the following existence results, as consequences of Theorem 3.1, Corollary 3.2 and Theorem 3.3, respectively.

For a given non-negative constant $c$ and a given positive constant $d$, with $\frac{2^{p} c}{(b-a)^{p-1} p M} \neq D_{2}(d)$, put

$$
b_{d}(c):=\frac{\|\alpha\|_{L^{1}([a, b])} G\left((p c)^{\frac{1}{p}}\right)-\|\alpha\|_{L^{1}([a+\alpha, b-\beta])} G(d)}{\frac{2^{p} c}{(b-a)^{p-1} p M}-D_{2}(d)},
$$

where

$$
D_{2}(d):=\int_{a}^{a+\alpha} H\left(\frac{d(p-1)(x-a)^{p-2}}{\alpha^{p-1}}\right) d x+\int_{b-\beta}^{b} H\left(-\frac{d(p-1)(b-x)^{p-2}}{\beta^{p-1}}\right) d x .
$$

Theorem 3.4. Assume that there exist a nonnegative constant $c_{1}$ and four positive constants $\alpha, \beta, c_{2}$ and $d$ with $\alpha+\beta<b-a$ and $c_{1}<\frac{(b-a)^{p-1} p M}{2^{p}} D_{2}(d)<c_{2}$ such that

(a) $\quad b_{d}\left(c_{2}\right)<b_{d}\left(c_{1}\right)$;

(a) $\quad g(0) \neq 0$ and there exist $v>p$ and $R>0$ such that for all $|t| \geq R$,

$$
0<v G(t) \leq \operatorname{tg}(t) \text {. }
$$

Then, for each $\lambda \in\left(\frac{1}{b_{d}\left(c_{1}\right)}, \frac{1}{b_{d}\left(c_{2}\right)}\right)$, problem (3.8) admits at least two positive classical solutions $u^{*}$ and $u^{* *}$, with $u^{*}$ such that

$$
\frac{2^{p} c_{1}}{(b-a)^{p-1} p M}<\int_{a}^{a+\alpha} H\left(u^{*}(x)\right) d x+\int_{b-\beta}^{b} H\left(u^{*}(x)\right) d x<\frac{2^{p} c_{2}}{(b-a)^{p-1} p M} .
$$

Remark 3.5. Since $\alpha(x) \geq 0$ a.e. $x \in[a, b], \alpha \neq \equiv 0, g: \mathbb{R} \longrightarrow \mathbb{R}$ is nonnegative and $h: \mathbb{R} \rightarrow] 0,+\infty[$ is positive, assuming $g(0) \neq 0$, and taking the strong maximum principle (see, for instance, [30, Theorem 11.1]) we observe that the solutions of the problem (3.8) are positive.

Corollary 3.6. Suppose that Assumption $\left(a_{6}\right)$ in Theorem 3.4 holds. Assume that there exist two positive constants $c$ and $d$ with $D_{2}(d)<\frac{2^{p} c}{(b-a)^{p-1} p M}$ such that

$$
\frac{G\left((p c)^{\frac{1}{p}}\right)}{c}<\frac{2^{p}\|\alpha\|_{L^{1}([a+\alpha, b-\beta])}}{(b-a)^{p-1} p M\|\alpha\|_{L^{1}([a, b])}} \frac{G(d)}{D_{2}(d)} .
$$

Then, for each $\lambda \in\left(\frac{D_{2}(d)}{\|\alpha\|_{L^{1}([a+\alpha, b-\beta])} G(d)}, \frac{\frac{2^{p} c}{(b-a)^{p-1} p M}}{\|\alpha\|_{L^{1}([a, b])} G\left((p c)^{\frac{1}{p}}\right)}\right)$, problem (3.8) admits at least two positive classical solutions $u^{*}$ and $u^{* *}$, with $u^{*}$ such that

$$
0<\int_{a}^{a+\alpha} H\left(u^{*}(x)\right) d x+\int_{b-\beta}^{b} H\left(u^{*}(x)\right) d x<\frac{2^{p} c}{(b-a)^{p-1} p M} .
$$


Theorem 3.7. Assume that there exist two positive constants $\bar{c}$ and $\bar{d}$ with $\frac{2^{p} \bar{c}}{(b-a)^{p-1} p M}<D_{2}(\bar{d})$ such that

(b5) $\quad \lim \sup _{|t| \rightarrow+\infty} \frac{G(t)}{|t|^{p}} \leq 0$.

Then, for each $\lambda>\overline{\lambda^{\prime}}$, where

$$
\overline{\lambda^{\prime}}=\frac{D_{2}(\bar{d})-\frac{2^{p} \bar{c}}{(b-a)^{p-1} p M}}{\|\alpha\|_{L^{1}([a+\alpha, b-\beta])} G(\bar{d})-\|\alpha\|_{L^{1}([a, b])} G\left((p \bar{c})^{\frac{1}{p}}\right)},
$$

problem (3.8) admits at least one positive classical solution $\bar{u}$ such that

$$
\int_{a}^{a+\alpha} H(\bar{u}(x)) d x+\int_{b-\beta}^{b} H(\bar{u}(x)) d x>\frac{2^{p} \bar{c}}{(b-a)^{p-1} p M} .
$$

Theorem 3.8. Assume that Assumption $\left(a_{6}\right)$ in Theorem 3.4 holds and

$$
\lim _{t \longrightarrow 0^{+}} \frac{g(t)}{|t|^{p-1}}=+\infty \text {. }
$$

Then, for $\lambda \in\left(0, \lambda^{*}\right)$, where $\lambda^{*}=\frac{\frac{2^{p}}{(b-a)^{p-1} p M}}{\|\alpha\|_{L^{1}([a, b])}} \sup _{c>0} \frac{c}{G\left((p c)^{\frac{1}{p}}\right)}$, problem (3.8) admits at least two positive classical solutions $u^{*}$ and $u^{* *}$, with $u^{*}$ such that

$$
0<\int_{a}^{a+\alpha} H\left(u^{*}(x)\right) d x+\int_{b-\beta}^{b} H\left(u^{*}(x)\right) d x<\frac{2^{p} c}{(b-a)^{p-1} p M} .
$$

Proof. For fixed $\lambda \in\left(0, \lambda^{*}\right)$, there exists a positive constant $c$ such that

$$
\lambda<\frac{\frac{2^{p} c}{(b-a)^{p-1} p M}}{\|\alpha\|_{L^{1}([a, b])} G\left((p c)^{\frac{1}{p}}\right)} .
$$

Moreover, by the condition (3.10), one has $\lim _{t \longrightarrow 0^{+}} \frac{G(t)}{|t|^{p}}=+\infty$. Therefore, we can choose a positive constant $d$ satisfying $D_{2}(d)<\frac{2^{p} c}{(b-a)^{p-1} p M}$ such that

$$
\frac{1}{\lambda\|\alpha\|_{L^{1}([a+\alpha, b-\beta])}}<\frac{G(d)}{D_{2}(d)} .
$$

Hence, from Corollary 3.6 we have the conclusion.

We here present the following example to illustrate the result of Theorem 3.8.

Example 3.9. Consider the problem

$$
\left\{\begin{array}{l}
-2\left|u^{\prime}(x)\right| u^{\prime \prime}(x)=\lambda e^{-x}\left(1+u^{8}\right)\left(2+\sin u^{\prime}\right), \quad x \in(0,1), \\
u(0)=u(1)=0
\end{array}\right.
$$

Let $\alpha(x)=e^{-x}$ for all $x \in[0,1], g(t)=1+t^{8}$ and $h(t)=1+\sin t$ for all $t \in \mathbb{R}$. It is clear that $\lim _{t \rightarrow 0^{+}} \frac{g(t)}{t^{2}}=$ $+\infty$ and Assumption $\left(\mathrm{a}_{6}\right)$ in Theorem 3.4 is satisfied. Note that $p=3$ and $M=3$, by applying Theorem 3.8, for every $\lambda \in\left(0, \frac{8}{9\left(1-e^{-1}\right)} \sup _{c>0} \frac{c}{\sqrt[3]{3 c}+3 c^{3}}\right)$ problem (3.11) has at least two positive classical solutions.

Now, by combining Corollary 3.6 and Theorem 3.7, we have the following theorem for the existence of three positive solutions for problem (3.8). 
Theorem 3.10. Assume that $g(0) \neq 0$ and

$$
\limsup _{|t| \longrightarrow+\infty} \frac{G(t)}{|t|^{p}} \leq 0
$$

Moreover, assume that there exist four positive constants $c, d, \bar{c}$ and $\bar{d}$ with

$$
\frac{(b-a)^{p-1} p M}{2^{p}} D_{2}(d)<c \leq \bar{c}<\frac{(b-a)^{p-1} p M}{2^{p}} D_{2}(\bar{d})
$$

such that (3.9) in Corollary 3.6, Assumption $\left(b_{4}\right)$ in Theorem 3.7, and

$$
\frac{G\left((p c)^{\frac{1}{p}}\right)}{c}<\frac{2^{p}}{(b-a)^{p-1} p M\|\alpha\|_{L^{1}([a, b])}} \frac{\|\alpha\|_{L^{1}([a+\alpha, b-\beta])} G(\bar{d})-\|\alpha\|_{L^{1}([a, b])} G\left((p \bar{c})^{\frac{1}{p}}\right)}{D_{2}(\bar{d})-\frac{2^{p} \bar{c}}{(b-a)^{p-1} p M}}
$$

are satisfied. Then, for each $\lambda \in \Lambda=\left(\max \left\{\overline{\lambda^{\prime}}, \frac{D_{2}(d)}{\|\alpha\|_{L^{1}([a+\alpha, b-\beta])} G(d)}\right\}, \frac{\frac{2^{p} c}{(b-a)^{p-1} p M}}{\|\alpha\|_{L^{1}([a, b])} G\left((p c)^{\frac{1}{p}}\right)}\right)$, where $\overline{\lambda^{\prime}}$ is given as in Theorem 3.7, problem (3.8) admits at least three positive classical solutions.

Proof. First, from (3.13), we see that $\Lambda \neq \emptyset$. Fix $\lambda \in \Lambda$. Using Corollary 3.6, we obtain the first positive solution $u^{*}$ with

$$
0<\int_{a}^{a+\alpha} H\left(u^{*}(x)\right) d x+\int_{b-\beta}^{b} H\left(u^{*}(x)\right) d x<\frac{2^{p} c}{(b-a)^{p-1} p M},
$$

which is a local minimum for the functional $\Phi-\lambda \Psi$. Theorem 3.7 guarantees the second positive solution with

$$
\int_{a}^{a+\alpha} H(\bar{u}(x)) d x+\int_{b-\beta}^{b} H(\bar{u}(x)) d x>\frac{2^{p} \bar{c}}{(b-a)^{p-1} p M} .
$$

Hence, using the mountain pass theorem as given by Pucci and Serrin ([12]), we have the desired result.

We now present the following consequence of Theorem 3.10.

Theorem 3.11. Assume that $g(0) \neq 0$,

$$
\limsup _{|t| \longrightarrow 0^{+}} \frac{G(t)}{|t|^{p}}=+\infty
$$

and

$$
\limsup _{|t| \longrightarrow+\infty} \frac{G(t)}{|t|^{p}}=0 .
$$

Furthermore, assume that there exist two positive constants $\bar{c}$ and $\bar{d}$ with $\frac{2^{p} \bar{c}}{(b-a)^{p-1} p M}<D_{2}(\bar{d})$ such that

$$
\frac{G\left((p \bar{c})^{\frac{1}{p}}\right)}{\bar{c}}<\frac{2^{p}\|\alpha\|_{L^{1}([a+\alpha, b-\beta])}}{(b-a)^{p-1} p M\|\alpha\|_{L^{1}([a, b])}} \frac{G(\bar{d})}{D_{2}(\bar{d})} .
$$

Then, for each

$$
\lambda \in\left(\frac{D_{2}(\bar{d})}{\|\alpha\|_{L^{1}([a+\alpha, b-\beta])} G(\bar{d})}, \frac{\frac{2^{p} \bar{c}}{(b-a)^{p-1} p M}}{\|\alpha\|_{L^{1}([a, b])} G\left((p \bar{c})^{\frac{1}{p}}\right)}\right),
$$

problem (3.8) admits at least three positive classical solutions. 
Proof. We easily see that (3.15) implies the condition $\left(\mathrm{b}_{5}\right)$ of Theorem 3.7. Moreover, by choosing $d$ small enough and $c=\bar{c}$, we observe that (3.9) in Corollary 3.6 follows from (3.14), as well as Assumption $\left(b_{4}\right)$ in Theorem 3.7, and (3.13) from (3.16). Hence, Theorem 3.10 yields the desired result.

Finally, as an example, we give the following result which is particular case of Theorem 3.11.

Theorem 3.12. Let $g: \mathbb{R} \rightarrow \mathbb{R}$ be a nonnegative continuous function such that $g(0) \neq 0$,

$$
\lim _{t \rightarrow 0^{+}} \frac{g(t)}{t}=+\infty \text { and } \lim _{t \rightarrow+\infty} \frac{g(t)}{t}=0 .
$$

Let $h: \mathbb{R} \rightarrow] 0,+\infty\left[\right.$ be a bounded and continuous function with $m:=\inf _{t \in \mathbb{R}} h(t)>0$ and $M:=\sup _{t \in \mathbb{R}} h(t)$ such that $\frac{8}{M}<D_{3}$, where $D_{3}:=\int_{0}^{8}\left(\int_{0}^{\tau} \frac{1}{h(\delta)} d \delta\right) d \tau-\int_{-8}^{0}\left(\int_{0}^{\tau} \frac{1}{h(\delta)} d \delta\right) d \tau$. Furthermore, suppose that

$$
\int_{0}^{\sqrt{2}} g(\xi) d \xi<\frac{4}{M D_{3}} \int_{0}^{2} g(\xi) d \xi
$$

Then, for each

$$
\lambda \in\left(\frac{D_{3}}{2 \int_{0}^{2} g(\xi) d \xi}, \frac{2}{M \int_{0}^{\sqrt{2}} g(\xi) d \xi}\right),
$$

the problem

$$
\left\{\begin{array}{l}
-u^{\prime \prime}(x)=\lambda g(u) h\left(u^{\prime}\right), \quad x \in(0,1), \\
u(0)=u(1)=0
\end{array}\right.
$$

admits at least three positive classical solutions.

Proof. Choosing $p=2, a=0, b=1, \alpha(x)=1$ for all $x \in[0,1], \bar{c}=1$, and $\bar{d}=2$, we observe that all hypotheses of Theorem 3.11 are satisfied. Hence the conclusion follows immediately.

\section{REFERENCES}

[1] R.P. Agarwal, H.B. Thompson, C.C. Tisdell, On the existence of multiple solutions to boundary value problems for second order ordinary differential equations, Dyn. Syst. Appl. 16 (2007), 595-609.

[2] M. Conti, S. Terracini, G. Verzini, Nehari's problem and competing species systems, Ann. Inst. H. Poincare Anal. Non Lineire 19 (2002), 871-888.

[3] D.G. Costa, C.A. Magalhaes, A variational approach to subquadratic perturbations of elliptic systems, J. Differential Equations 111 (1994), 103-122.

[4] J.R. Graef, S. Heidarkhani, L. Kong, A critical points approach for the existence of multiple solutions of a Dirichlet quasilinear system, J. Math. Anal. Appl. 388 (2012), 1268-1278.

[5] D.D. Hai, R. Shivaji, An existence result for a class of $p$-Laplacian systems, Nonlinear Anal. 56 (2004), 1007-1010.

[6] D.D. Hai, H. Wang, Nontrivial solutions for p-Laplacian systems, J. Math. Anal. Appl. 330 (2007), 186-194.

[7] S. Heidarkhani, J. Henderson, Multiple solutions for a Dirichlet quasilinear system containing a parameter, Georgian Math. J. 21 (2014), 187-197.

[8] S. Heidarkhani, Y. Tian, Multiplicity results for a class of gradient systems depending on two parameters, Nonlinear Anal. 73 (2010), 547-554.

[9] A. Kristály, D. Repovš, Multiple solutions for a Neumann system involving subquadratic nonlinearities, Nonlinear Anal. 74 (2011), 2127-2132.

[10] J. Zhang, Z. Zhang, Existence results for some nonlinear elliptic systems, Nonlinear Anal. 71 (2009), $2840-2846$.

[11] B. Ricceri, A three critical points theorem revisited, Nonlinear Anal. 70 (2009), 3084-3089.

[12] P. Pucci, J. Serrin, A mountain pass theorem, J. Differential Equations 60 (1985), 142-149.

[13] G.A. Afrouzi, S. Heidarkhani, Three solutions for a quasilinear boundary value problem, Nonlinear Anal. 69 (2008), 3330-3336. 
[14] D. Averna, G. Bonanno, Three solutions for quasilinear two-point boundary value problem involving the one-dimensional p-Laplacian, Proc. Edinburgh Math. Soc. 47 (2004), 257-270.

[15] S. Heidarkhani, J. Henderson, Critical point approaches to quasilinear second order differential equations depending on a parameter, Topol. Methods Nonlinear Anal. 44 (2014), 177-197.

[16] R. Livrea, Existence of three solutions for a quasilinear two point boundary value problem, Arch. Math. 79 (2002), 288298.

[17] G. Bonanno, S. Heidarkhani, D. O’Regan, Nontrivial solutions for Sturm-Liouville systems via a local minimum theorem for functionals, Bull. Aust. Math. Soc. 89 (2014), 8-18.

[18] S. Heidarkhani, Existence of non-trivial solutions for systems of $n$ fourth order partial differential equations, Math. Slovaca 64 (2014), 1249-1266.

[19] G. D'Aguì, Multiplicity results for nonlinear mixed boundary value problem, Bound. Value Probl. 2012 (2012), Article ID 134.

[20] J.R. Graef, S. Heidarkhani, L. Kong, Nontrivial periodic solutions to second-order impulsive Hamiltonian systems, Electron. J. Differential Equations 2015 (2015), Article ID 204.

[21] S. Heidarkhani, M. Ferrara, S. Khademloo, Nontrivial solutions for one-dimensional fourth-order Kirchhoff-type equations, Mediterr. J. Math. 13 (2016), 217-236.

[22] J.R. Graef, S. Heidarkhani, L. Kong, A variational approach to a Kirchhoff-type problem involving two parameters, Results Math. 63 (2013), 877-889.

[23] S. Heidarkhani, G.A. Afrouzi, A. Hadjian, Multiplicity results for elliptic problems with variable exponent and nonhomogeneous Neumann conditions, Math. Meth. Appl. Sci. 38 (2015), 2589-2599.

[24] S. Heidarkhani, M. Ferrara, G.A. Afrouzi, G. Caristi, S. Moradi, Existence of solutions for Dirichlet quasilinear systems including a nonlinear function of the derivative, Electron. J. Differential Equations 2016 (2016), Article ID 56.

[25] S. Heidarkhani, A. Salari, $p(x)$-Laplacian-like problems with Neumann condition originated from a capillary phenomena, J. Nonlinear Funct. Anal. 2018 (2018), Article ID 11.

[26] G. Bonanno, A critical point theorem via the Ekeland variational principle, Nonlinear Anal. 75 (2012), 2992-3007.

[27] B. Ricceri, A general variational principle and some of its applications, J. Comput. Appl. Math. 113 (2000), $401-410$.

[28] G. Talenti, Some inequalities of Sobolev type on two-dimensional spheres, W. Walter (Ed.), General Inequalities, Internat. Schriftenreihe Numer. Math., vol. 5 (1987), pp. 401-408.

[29] P.H. Rabinowitz, Minimax methods in critical point theory with applications to differential equations, CBMS Regional Conference Series in Mathematics, vol. 65. AMS, Providence, RI (1986)

[30] A. Ambrosetti, P. Rabinowitz, Dual variational methods in critical points theory and applications, J. Funct. Anal. 14 (1973), 349-381. 